\title{
From the Editors
}

This issue of the TESL Canada Journal stretches from coast to coast. There are articles stemming from Newfoundland and British Columbia (plus many points in between). We are delighted to be able to present such a wide range of material, as we feel it is one of the major roles of the Journal to allow members of TESL Canada from all across the country (and indeed all around the world) to share their ideas with one another. Only by maintaining contact can we foster the sense of community, the sense of connection, and the ability to grow that is so crucial to any professional group. We feel this is one of the most beneficial aspects of a national organization like TESL Canada. For upon reading all of these articles, we were struck by how much we can learn from the research, reflections, and concerns of people all over Canada. Even where regional concerns are specifically addressed (e.g. Tonya Bassler on refugee concerns in Newfoundland) there is very definitely a message to TESL professionals in every other province and territory.

We are all concerned with communication; it's our business and it's our pleasure. The lines of communication amongst ourselves are vital. We are proud of the role we play at the Journal in keeping these lines open, but, of course, without the provincial members' support of TESL Canada none of this would be possible. Our national organization takes its strength from you its members. From coast to coast.

\section{David Mendelsohn and John Archibald Editors}




\section{Un mot des Editeurs}

Dans ce numéro de la revue "TESL CANADA" nous parcourons le Canada d'un océan à l'autre. Il y a des articles qui proviennent des provinces de TerreNeuve et de la Colombie britannique (en passant par plusieurs points entre les deux). Nous sommes heureux de pouvoir présenter un aussi grand nombre d'études et d'analyses, car nous croyons qu'un de nos rôles principaux est de permettre à tous nos membres venant de partout au Canada (et du monde entier) de partager leurs idées entre eux. C'est seulement en maintenant un contact que nous pouvons favoriser le sans de la communauté, le sans de l'inter-relation et la capacité de croître qui est si cruciale à tout groupe professionnel. Nous croyons que c'est un des aspects les plus avantageux d'une organisation nationale, comme "TESL CANADA".

C'est ainsi qu'à la suite de la lecture de tous ces articles nous avons été frappés par la quantité considérable de connaissances que nous pouvons acquèrir à partir des recherches, réflexions et préoccupations des gens d'un bout à l'autre du Canada. Même, en présentant des préoccupations d'ordre régional (comme par exemple, l'étude de Tonya Bassler sur les préoccupations des réfugiés à TerreNeuve) nous transmettons sûrement un message aux professionnels de "TESL" dans chacune des autres provinces et territoires.

Nous sommes tous intéressés par la communication, nous en faisons notre profession et nous en tirons beaucoup de satisfaction. Les voies de la communication sont vitales pour nous tous. Nous sommes fiers du rôle que nous jouons à la revue "TESL CANADA" en permettant de maintenir ces voies ouvertes, mais sans l'appui de nos membres rien ne serait possible. Notre organisation nationale tire ses forces de vous, les membres d'un océan à l'autre.

David Mendelsohn and John Archibald

Editeurs 